\title{
Human Middle Temporal Cortex, Perceptual Bias, and Perceptual Memory for Ambiguous Three-Dimensional Motion
}

\author{
Jan W. Brascamp, ${ }^{1}$ Ryota Kanai, ${ }^{2}$ Vincent Walsh, ${ }^{2}$ and Raymond van Ee ${ }^{1}$ \\ ${ }^{1}$ Helmholtz Institute, Utrecht University, 3584 CH, Utrecht, The Netherlands, and ${ }^{2}$ Insitute of Cognitive Neuroscience, University College London, London \\ WC1N 3AR, United Kingdom
}

When faced with inconclusive or conflicting visual input human observers experience one of multiple possible perceptions. One factor that determines perception of such an ambiguous stimulus is how the same stimulus was perceived on previous occasions, a phenomenon called perceptual memory. We examined perceptual memory of an ambiguous motion stimulus while applying transcranial magnetic stimulation (TMS) to the motion-sensitive areas of the middle temporal cortex (hMT + ). TMS increased the predominance of whichever perceptual interpretation was most commonly reported by a given observer at baseline, with reduced perception of the less favored interpretation. This increased incidence of the preferred percept indicates impaired long-term buildup of perceptual memory traces that normally act against individual percept biases. We observed no effect on short-term memory traces acting from one presentation to the next. Our results indicate that hMT + is important for the long-term buildup of perceptual memory for ambiguous motion stimuli.

\section{Introduction}

The human brain displays a high degree of adaptability. In the visual system, numerous phenomena, such as priming, adaptation, and learning, testify to the dynamic adjustment of neural processing to input and task requirements (Kristjánsson, 2006; Schwartz et al., 2007).

The present work centers on a form of history dependence in vision (Leopold et al., 2002) that has been referred to as perceptual memory (Maier et al., 2003), a term commonly applied to phenomena at the intersection of mnemonic and sensory function (Schacter and Buckner, 1998; Magnussen and Greenlee, 1999). Here, observers view ambiguous input that may be perceived in one of a number of ways. A Necker cube, for example, can be seen as a cube in either of two configurations. Perceptual memory in the sense adopted here refers to the fact that perception of such ambiguous input upon renewed presentation depends, in part, on what was perceived during previous encounters with the same input.

Perceptual memory has a short-acting component that has been described as a type of priming or retention spanning from one encounter with a stimulus to the next (Noest et al., 2007). This aspect of perceptual memory is naturally expressed as the tendency for perception on a given presentation with an ambig-

Received Aug. 24, 2009; revised 0ct. 9, 2009; accepted 0ct. 26, 2009.

This work was supported by the British Council Partnership Programme in Science/Platform Bèta Techniek (J.W.B.), the Human Frontiers Science Program (R.K.), the Medical Research Council (V.W.), and a High Potential grant from Utrecht University (R.v.E.). We are grateful to Randolph Blake for providing valuable comments on a previous version of this manuscript.

Correspondence should be addressed to Jan W. Brascamp at his present address: Vanderbilt University, Department of Psychology, 111 21st Avenue South, Nashville, TN 37203. E-mail:j.w.brascamp@gmail.com.

DOI:10.1523/JNEUROSCI.4171-09.2010

Copyright $\odot 2010$ the authors $\quad 0270-6474 / 10 / 300760-07 \$ 15.00 / 0$ uous stimulus to conform to perception on the immediately preceding encounter. Perceptual memory also has longer-acting components that accumulate over many repetitions of the same stimulus (Maloney et al., 2005; Brascamp et al., 2008, 2009a; Pastukhov and Braun, 2008). These accumulating traces are responsible for the evolution of a slow perceptual alternation cycle when the same ambiguous stimulus is shown repeatedly, leading the alternative interpretations to take turns dominating perception from tens to hundreds of consecutive presentations (Brascamp et al., 2009a).

Numerous characteristics of perceptual memory have now been described and various computational models developed to understand its mechanisms (for review, see Pearson and Brascamp, 2008). Several of these efforts have converged on the notion that perception of an ambiguous stimulus alters response characteristics of the associated representations within sensory cortex (Chen and He, 2004; Pearson and Clifford, 2004). Upon renewed presentation of the same stimulus, this altered response pattern influences competitive interactions that occur between alternative perceptual interpretations. Nonsensory, frontal, and parietal brain areas have also been implicated in perceptual memory (Sterzer and Rees, 2008). However, direct measurements of perceptual memory related neural activity are scarce and, as yet, inconclusive with regard to whether sensory or extrasensory mechanisms predominate.

In this study observers report perception of a dynamic stimulus that is ambiguous with respect to three-dimensional rotation direction. We present the same stimulus repeatedly and examine the effects on perceptual memory of transcranial magnetic stimulation (TMS) applied to the complex of motion-sensitive areas in the human middle temporal cortex $(\mathrm{hMT}+)$. These are sensory areas implicated in the perception of this type of stimuli 


\section{A One session}

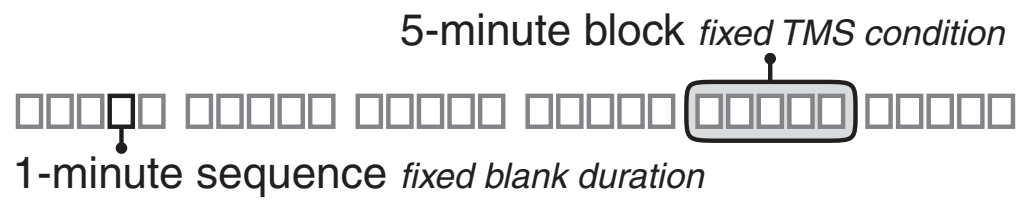

time

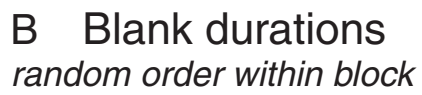

\section{TMS conditions random order between blocks}
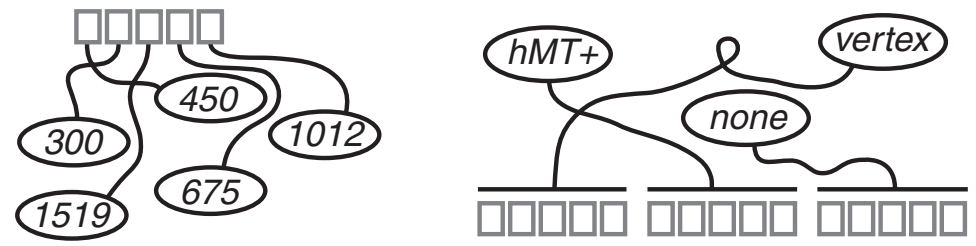

Figure 1. Trial structure. $\boldsymbol{A}$, Thirty minute sessions were separated into six 5 min blocks, within which the TMS condition remained fixed. Each block, in turn, consisted of five 1 min sequences during which the stimulus was intermittently presented with a fixed blank duration between presentations. $\boldsymbol{B}$, Blank durations, five in total, were distributed in random order across the five sequences of one block. $C$, The three TMS conditions were distributed in random order across the first three blocks of a session, and again in random order across the last three blocks.

(Grunewald et al., 2002; Parker et al., 2002; Brouwer and van Ee, 2007), rendering them a natural choice considering the possible sensory nature of this memory.

Part of this work was previously submitted as a conference abstract to the 2009 annual meeting of the Vision Sciences Society in Naples, Florida (Brascamp et al., 2009b).

\section{Materials and Methods}

Experimental procedure. Observers were seated in a darkened room in front of a computer screen with their heads stabilized on a chin and forehead rest. They viewed a structure-from-motion stimulus (see below) that was ambiguous with respect to its direction of rotation. The stimulus was presented intermittently during short ( $1 \mathrm{~s})$ presentations separated by blank intervals. Observers were instructed to maintain fixation in the middle of the display and to report rotation direction on every presentation. Individual presentations were short enough to minimize perceived direction reversals during presentation. Every observer completed one session of $30 \mathrm{~min}$ (Fig. 1A), separated into six blocks of $5 \mathrm{~min}$, within each of which we applied a single TMS condition. Every 5 min block, in turn, consisted of five 1 min sequences of turning the stimulus on and off at a steady pace, keeping the blank duration between consecutive presentations fixed within each 1 min sequence.

We did not monitor eye movements during this experiment, but eye movements are unlikely to have contributed importantly to our results. TMS effects on eye movements are not uncommon, but are usually associated with stimulating other brain areas than we did, like the frontal eye fields, and only if eye movement preparation or execution falls within a relatively narrow time window (up to $150 \mathrm{~ms}$ ) around the TMS pulse (Gagnon et al., 2006; Drew and Donkelaar, 2007; Walker et al., 2009). In our case, visual stimulation did not start until several hundred milliseconds after TMS was applied, and then lasted for another second (see below). This renders a perceptual effect of TMS via eye movements unlikely. Moreover, whether eye movement modulations in general influence perception of our stimulus is questionable. Previous studies have found no consistent relationship between eye movements and perception of ambiguous stimuli very similar to ours (Brouwer and van Ee, 2006, 2007; Klink et al., 2008), and a recent study that did observe such a relation (using a different ambiguous stimulus) showed that, never- theless, manipulating eye movements did not influence perception (Laubrock et al. 2008).

Observers. Nine neurologically healthy observers participated: one author and eight paid subjects who were naive as to the purpose of the experiment. Informed written consent was obtained beforehand, and the experiment was approved by the local ethics committee at University College London.

Visual stimulus. Stimuli were presented on a $34 \times 25.5 \mathrm{~cm}$ gamma-linearized cathode ray tube monitor positioned at $57 \mathrm{~cm}$ from the observer. The resolution and refresh rate were $1024 \times 768$ pixels and $75 \mathrm{~Hz}$, respectively. The visual stimulus consisted of 200 yellow random dots with unlimited lifetime, moving back and forth horizontally on a black background. The luminance of the dots and the background was 94 and $0.5 \mathrm{~cd} / \mathrm{m}^{2}$, respectively. The dots' speed profile mimicked that of a flat projection of dots scattered on the surface of a transparent globe revolving around its central vertical axis (i.e., a sinusoidal speed profile). This type of display readily elicits the illusion of the full, three-dimensional, object (Wallach and O'Connell, 1953). Due to a lack of additional depth cues indicating which motion direction corresponded to the front, and which to the back surface of the globe, observers alternately perceived either rotation direction (Braunstein, 1977). Less commonly, the same stimulus may in some cases be perceived as two "half-spheres" that both point outward toward the observer while sliding in opposite directions, one behind the other (Hol et al., 2003). Although our observers did not spontaneously report this perception, we preempted any confusion it might cause by instructing observers to report the motion direction of the surface perceived to be in front, regardless of whether the hind surface was convex or concave. The stimulus radius was $1.7^{\circ}$ and the simulated rotation speed was 0.9 revolutions/s. The average dot density within the circular area covered by the simulated globe was 22 dots per square degree of visual angle. At each stimulus onset the dots started from a different, randomly drawn position. A small yellow cross $\left(0.17 \times 0.17^{\circ}\right)$ marked fixation in the center of the display. Each 5 min block of a given TMS condition was separated into five sequences of $1 \mathrm{~min}$, during each of which the stimulus was switched on and off at a steady pace with presentation duration fixed at $1 \mathrm{~s}$ and the blank duration between consecutive presentations set to either $300,450,675,1012$, or 1519 ms. These five blank durations were randomly assigned to the five sequences of each 5 min block (Fig. $1 B$ ). We chose this range of blank durations because the effects of perceptual memory change rapidly within this range, thus avoiding ceiling effects that might mask a potential influence of TMS in timing regimes where perceptual memory is more unvarying. Within the particular timing range we chose, longer blank durations tend to cause repetition of the same percept on consecutive presentations, whereas shorter blank durations are less likely to yield percept repetition and in some cases even tend to cause opposite perception on consecutive presentations (Orbach et al., 1966; Noest et al., 2007; Klink et al., 2008).

TMS procedure. We applied three TMS conditions: TMS over hMT+, no TMS (baseline), and TMS over the vertex (control for nonspecific effects of TMS such as tactile and acoustic artifacts). The three conditions were applied in random order during the first 3 blocks of each session, and again in random order during the last 3 blocks (Fig. 1C). Transcranial magnetic stimulation was applied using a $70 \mathrm{~mm}$ figure-eight coil connected to a MagStim SuperRapid TMS machine, set to $60 \%$ of maximal machine output level. During TMS blocks a brief train of three pulses, spaced $40 \mathrm{~ms}$ apart, was applied at the midpoint of every third blank interval. For instance, when the blank interval was $300 \mathrm{~ms}$ long, the three pulses fell 110, 150, and 190 ms into the blank interval. We 
chose this short interval between pulses to leave a temporal gap between the pulse train and the presentations that preceded and followed it (a $110 \mathrm{~ms}$ gap at the shortest blank duration of $300 \mathrm{~ms}$ ), thereby minimizing the direct interaction between our pulses and the visual stimulation itself (O'Shea et al., 2004). Our motivation for stimulating only every third blank interval was twofold: to be able to compare perception at various moments relative to the pulse train, and not to exceed safety standards of TMS stimulation at the shortest blank duration (Wassermann, 1998).

Right hMT + was localized in individual observers using the Brainsight neuronavigation system (Rogue Research). To this end observers' structural magnetic resonance imaging (MRI) scans were transformed into standard coordinates using FSL (FMRIB Software), after which the coordinates of hMT + in standard space, taken from the literature, were transformed to the individual brains using the inverse transformation. For standard coordinates we used $(x, y, z)=(49,-67.25$,

$0.5)$, obtained by averaging the coordinates reported in four existing studies (Dumoulin et al., 2000; Sterzer et al., 2002; Campana et al., 2006; Sterzer and Kleinschmidt, 2007). Correct localization of hMT+ was verified by visual inspection of its position relative to anatomical landmarks on the structural MRI scans (Dumoulin et al., 2000). For reference, Figure 2 depicts a structural brain scan of one representative observer, displaying both the hMT + location we obtained based on standard coordinates and the relevant anatomical landmarks. In general, the correspondence between various methods of hMT + localization in the literature is good. For instance, the location of hMT + as determined using TMS on the basis of phosphenes corresponds well with the locations of the anatomical landmarks we used for reference (Stewart et al., 1999; Théoret et al., 2002). Conversely, $\mathrm{hMT}+$ localization using anatomical landmarks results in coordinates that correspond well to those obtained when mapping hMT+ using functional brain imaging, and reliably results in phosphene generation when applying TMS (Campana et al., 2006).

We chose to stimulate hMT + only in the right hemisphere because there is no evidence or hypothesis for lateralization of memory functions in hMT + and, in addition, because receptive fields in this brain region tend to cross the vertical meridian (Dukelow et al., 2001; Huk et al., 2002), obviating the need for lateral controls given our small and central visual stimulus. During stimulation of hMT + the coil was positioned parallel to the scalp, with the handle pointing dorsally and $\sim 45^{\circ}$ upward. For vertex stimulation we positioned the coil halfway between the inion and the nasion, and halfway between the two ears, and oriented it parallel to the scalp with the handle pointing dorsally.

\section{Results}

We examined two measures of perceptual memory: (1) the oneback effects of the immediately preceding presentation and (2) the cumulative effects of repeated exposures. We observed an effect of hMT + TMS on the latter, but not the former measure.

\section{One-back effect}

A straightforward expression of perceptual memory is the tendency for perception on a given encounter with a particular ambiguous stimulus to conform to perception on the previous encounter-a form of priming (Leopold et al., 2002; Maier et al., 2003; Noest et al., 2007). This tendency is particularly robust at longer blank durations (multiple seconds), and reduced or even reversed at short blank intervals, near the lower end of the range we measured (Noest et al., 2007; Klink et al., 2008). Our first index of perceptual memory is therefore simply the proportion of presentations on which the perceived rotation direction is equal to that on the preceding presentation.

Figure $3 A$ shows this measure for the five blank durations we applied ( $x$-axis), and for our three TMS conditions (different shades). The position of these curves above a proportion of 0.5 (two-tailed paired $t$ test combining across TMS conditions, $p<$ 0.01 ) indicates a tendency for perception on consecutive presentations to repeat. Moreover, we observe an increased proportion of percept repetition as the duration of the blank phase increases (ANOVA with factors observer, blank duration, TMS condition gives $p<0.01$ for blank duration and, after averaging over observers and conditions, Spearman rank correlation gives $\rho=1$ and $p=$ 0.02). These findings replicate previous work (Leopold et al., 2002; Maier et al., 2003; Noest et al., 2007; Klink et al., 2008), thus confirming we replicated the perceptual memory phenomenon.

Figure $3 A$ does not, however, show any difference in the proportion of percept repetition between blocks with different TMS conditions (same three-way ANOVA; $p=0.21$ for the factor TMS condition). This is confirmed in Figure $3 B$, where the same data have been collapsed across blank duration (two-tailed paired $t$ test; $p=0.26$ for hMT + TMS vs no TMS, and $p=0.64$ for hMT + TMS vs vertex TMS).

During TMS blocks we applied transcranial stimulation only during every third blank interval (see Materials and Methods). We therefore repeated the analyses of Figure 3 separately for presentations that occurred at various moments relative to the pulse train (i.e., directly after, two presentations later or three presentations later). This again yielded no significant effect of TMS for any of the moments relative to the pulse train.

\section{Cumulative effect}

The second variable we investigated was the cumulative effect of perceptual memory. Rather than only a form of priming that acts over a relatively short term, perceptual memory may build up throughout an experimental session (Brascamp et al., 2008; Pastukhov and Braun, 2008) and may, over the course of that session, exhibit suppressing effects (i.e., a negative dependence on prior perception) instead of its priming effects (Brascamp et al., 2009a). During prolonged sessions of intermittent presentation of an ambiguous stimulus this accumulation ultimately leads to the evolution of a slow perceptual alternation cycle during 


\section{Same percept reported on two consecutive presentations}

A

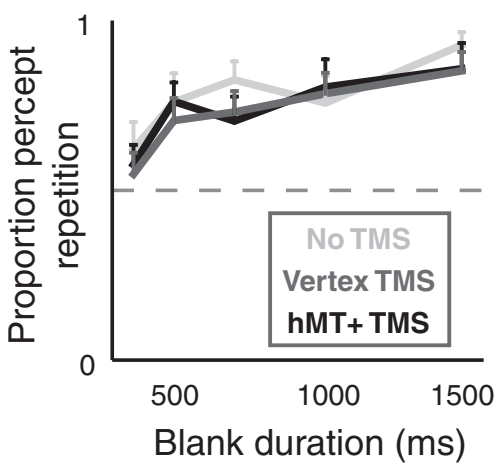

B

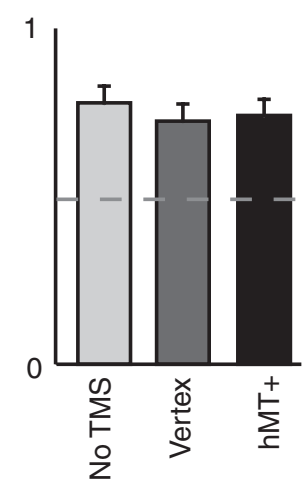

Figure 3. Percept repetition. $\boldsymbol{A}$, The proportion of pairs of consecutive presentations on which the same percept was repeated twice ( $y$-axis) as a function of blank duration ( $x$-axis), across all nine observers. There is no significant difference between the three TMS conditions. $\boldsymbol{B}$, The same data collapsed across blank durations. Error bars are SEs of the sample mean; only upward bars shown to avoid clutter.

which the alternative interpretations take turns dominating perception on (almost) every presentation for minutes at a time (Brascamp et al., 2009a).

To measure the cumulative effect of perceptual memory we made use of the known interaction between this effect and individual percept bias. Perception of ambiguous stimuli is influenced, not only by perceptual memory of prior encounters, but also by an observer's individual bias toward a particular percept (their "preferred" percept) (Carter and Cavanagh, 2007; Knapen et al., 2009). This bias is not noticeably dependent on prior perception and remains stable across sessions separated by weeks (Carter and Cavanagh, 2007). This percept bias is especially prominent during short experimental sessions (minutes) (Carter and Cavanagh, 2007). It is much weaker, however, during longer sessions (tens of minutes) that allow the evolution of the slow perceptual alternation cycle caused by perceptual memory accumulation, which is fairly balanced in terms of relative prevalence of the alternative interpretations (Brascamp et al., 2009a). In other words, because of perceptual memory the predominance of the preferred percept gradually reduces over the course of an experimental session (see Brascamp et al., 2008, their supplemental Fig. S1), as perceptual memory accumulates and initiates a slow and balanced perceptual alternation cycle. Below we make use of this property, by using the predominance of the preferred percept as an index of perceptual memory accumulation.

We first verified that our experiment replicated the described interaction between observer bias and counteracting memory accumulation. By means of illustration, Figure $4 A$ displays perception as reported by one observer during a typical 30 min session (containing various TMS conditions and blank durations). It gives an impression, both of the initial predominance of that observer's preferred percept (rightward rotation), and of the more balanced cycle of alternating perception that takes hold as a session progresses. Figure $4 B$ shows in a more systematic manner how the influence of percept bias evolved during sessions of all our observers. The proportion of presentations on which observers reported their preferred percept (identified as the percept that a given observer reported the most overall) is plotted as a function of time within a session (averaging within a 10 min sliding
A
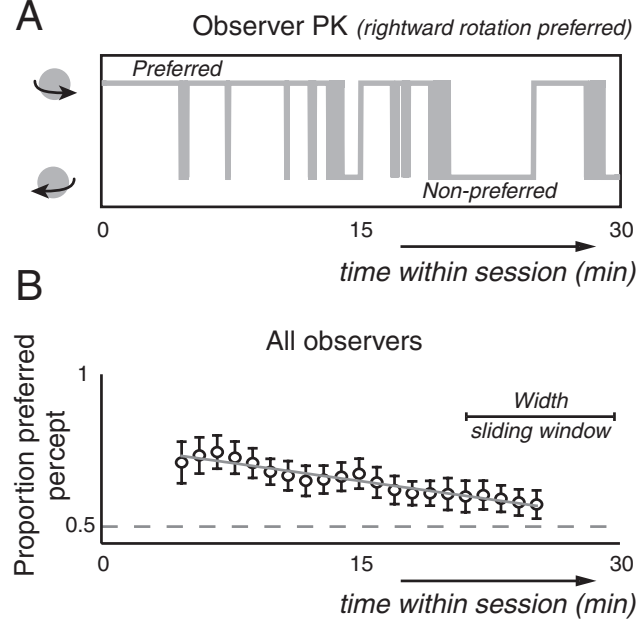

Figure 4. Perceptual sequence throughout a session. $\boldsymbol{A}$, Example of a perceptual sequence. Perceived rotation direction ( $y$-axis) as reported by one observer over the course of one experimental session ( $x$-axis). This example illustrates key features of the perceptual sequence during a session. Observers generally started off perceiving predominantly their preferred percept (rightward rotation in this case) for minutes on end. Further into the experiment they often started to experience a slow cycle of alternating perception, with each percept predominating more or less exclusively for minutes at a time, separated by more unstable periods featuring rapid alternations between the competing interpretations. Perception is drawn as continuous lines for clarity, even though blank intervals separated individual presentations. $\boldsymbol{B}$, Predominance of the preferred percept throughout sessions. The proportion of dominance of the preferred percept is plotted as a function of time within a session, averaged across all observers. The proportion systematically decreases as a session progresses, indicative of accumulating perceptual memory gradually supplanting percept bias as the main determinant of perception. Error bars are SEs of the sample mean. Data were averaged within a 10 min sliding window, with the $x$-axis representing the center of the window (no data are shown for the first and last $5 \mathrm{~min}$ in this plot because there the sliding window falls outside of the session).

window). The predominance of the preferred percept systematically decreases over the course of a session (Spearman, $\rho=-0.33$ and $p<0.01$ ), replicating previous findings (Brascamp et al., 2008), and indicating that the described interaction between accumulating perceptual memory traces and stationary bias holds for our experiment.

This accumulation of perceptual memory traces, which reduces the influence of individual percept bias, is clearly affected by TMS to hMT + . Figure $5 A$ shows the proportion of presentations on which observers reported their preferred percept, identified as the percept that a given observer reported the most overall (we also confirmed this by defining the preferred percept as that reported most during the first $10 \mathrm{~min}$ of each session and found the same effects). This proportion depends significantly on TMS condition (three-way ANOVA with factors observer, TMS condition and blank duration gives $p<0.01$ for the factor TMS condition), with a significantly increased incidence of the preferred percept in the hMT + TMS condition relative to the no TMS condition (two-tailed paired $t$ test, $p=0.02$ ) and relative to the vertex TMS condition $(p<0.01)$. The incidence of the preferred percept does not depend significantly on the blank duration (same three-way ANOVA, $p=0.38$ ), nor does the effect of TMS vary with blank duration (TMS condition $\times$ blank duration $p=0.56)$.

Further evidence that hMT+ TMS interferes with the accumulation of perceptual memory is presented in Figure $5 B$. Here, we plot the prevalence of the preferred percept as a function of sequence number within each block. Recall that our sessions were built up of 5 min blocks for each TMS condition, each of which 


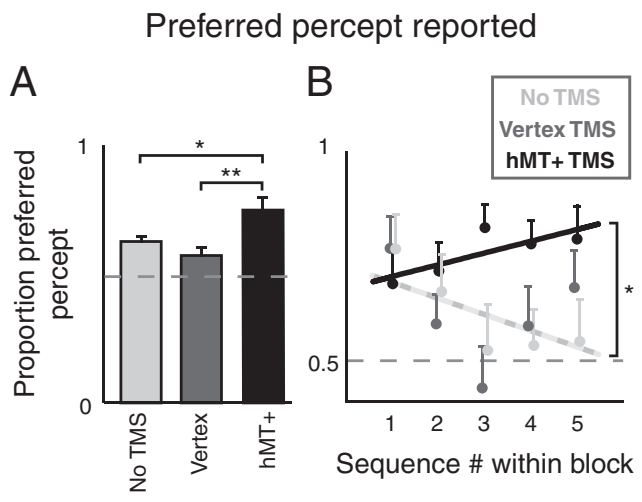

Figure 5. The preferred percept, i.e., the overall more predominant percept for a given observer. $\boldsymbol{A}$, The proportion of presentations on which the preferred percept was experienced, collapsed across blank durations. The preferred percept dominates more during blocks of hMT + TMS than during other blocks, consistent with weaker perceptual memory in that condition. $\boldsymbol{B}$, The proportion of dominance of the preferred percept as a function of the sequence number within a block with a given TMS condition (each block consisted of five such sequences) (Fig. 1). The proportion systematically falls from sequence to sequence within blocks with no TMS or vertex TMS (cf. Fig. $4 B$ ), but not during blocks of hMT + TMS, indicative of a disruption of memory buildup. Error bars are SEs of the sample mean; only upward bars shown to avoid clutter. Lines are fitted regression lines.

consisted of five 1 min sequences of presentations (Fig. 1). If TMS does interfere with the accumulation of perceptual memory, the overall downward trend in the predominance of the preferred percept over the course of a session (Fig. $4 \mathrm{~B}$ ) may be more obvious in some 5 min blocks than others, dependent on the TMS condition within a given block. Our data support this prediction: the proportion preferred perception depends differently on sequence number for different TMS conditions (threeway ANOVA with factors observer, TMS condition and sequence number; interaction TMS condition $\times$ sequence number gives $p=0.03$ ). Indeed, within blocks during which we applied either no TMS (light gray dots) or vertex TMS (darker gray dots) the proportion of preferred perception significantly decreased from one sequence to the next (Spearman, $\rho=-0.15$ and $p=0.03$; dashed regression line), consistent with the overall decrease over time observed throughout a session (Fig. $4 B$ ). In contrast, during blocks of hMT + TMS the incidence of the preferred percept is not significantly reduced from one sequence to the next (Spearman, $\rho=0.12$ and $p=0.12$ ). Indeed, we even observe an upward trend in this condition (black regression line), suggestive of a degradation of perceptual memory during these blocks. (Note that for the analyses accompanying Fig. $5 B$ we pooled the No TMS and Vertex TMS conditions, after assuring no significant difference between these two conditions $(p=0.97)$ nor between their interactions with sequence number ( $p=0.66$, three-way ANOVA with conditions observer, TMS condition, and sequence number.)

The effects shown in Figure 5 are not due to the increased incidence of one particular perceived rotation direction (e.g., rightward), as percept preference was balanced across our observers ( 4 observers had a leftward bias; 5 had a rightward bias). Indeed, repeating the analysis of Figure 5 for the rightward or leftward percept rather than for each observer's individual preferred percept does not yield any of the described effects. In addition, the increased incidence of the preferred percept during hMT + TMS blocks cannot be explained by those blocks being presented earlier within our sessions on average than other blocks, due to a fluke outcome of the randomization procedure. Numbering the six blocks within our sessions chronologically from one to six, the average positions of No TMS, Vertex TMS, and hMT+ TMS blocks were 3.6, 3.4, and 3.5, respectively: close to the expected value of 3.5 (the midpoint of the session) and not different for the hMT + blocks, showing they did not occur earlier or later than the other blocks on average.

Even though we administered the TMS pulses during the blank intervals between stimulus presentations, one may consider whether the observed effects of hMT + TMS could have to do with a direct interference with sensory processing of our moving stimulus. Previous work has shown such interference of hMT + TMS with motion processing, as witnessed by impaired motion detection and discrimination (Hotson et al., 1994; Théoret et al., 2002; Silvanto et al., 2005; Laycock et al., 2007; McKeefry et al., 2008), as well as increased reaction times to moving stimuli (Walsh et al., 1998; Ellison et al., 2003). In our experiment, such interference with motion processing could indirectly reduce buildup of perceptual memory by impairment of sensory signals that feed into a putative memory locus. In the extreme case, one could even conceive of TMS degrading observers' perception of motion in depth to a point where their task becomes one of near-threshold direction discrimination (cf. Salzman et al., 1992), potentially explaining why they report their preferred percept more often (although it should be noted that, during debriefing, none of our observers reported perceiving anything but rotating sphere surfaces). To test for any direct effects of TMS on sensory processing in our experiments, we therefore investigated whether observers gave their reports any less reliably or any less quickly in the hMT+ condition compared with the other conditions. This analysis yielded no evidence that any direct interference with sensory processing occurred: the proportion of failures to respond was as low in the hMT + TMS condition as in other conditions $(2.0,2.2$, and $2.0 \%$ of presentations missed in the no TMS, vertex TMS, and hMT+ TMS condition, respectively; three-way ANOVA with factors observer, blank duration, and TMS condition, $p=0.86$ for the factor TMS condition), and the response latency after stimulus onset was also unaffected (564, 579, and $590 \mathrm{~ms}$ in the no TMS, vertex TMS, and hMT+ TMS condition, respectively; $p=0.11$ ).

\section{Discussion}

Our findings show that hMT + plays an important role in determining the outcome of the perception of ambiguous structurefrom-motion and that its role is based on representational changes that occur over repeated exposures to the same stimulus, i.e., cumulative perceptual memory. These results extend previous findings of a role for hMT + in motion priming (Campana et al., 2002, 2006) and for its presumptive macaque homolog in short-term motion memory (Bisley et al., 2001). They furthermore fit well with evidence that various kinds of priming and memory in the sensory domain draw on mechanisms within sensory cortex itself (Schacter and Buckner, 1998; Magnussen and Greenlee, 1999; Pasternak and Greenlee, 2005, Pearson and Brascamp, 2008; Sterzer and Rees, 2008).

The demonstration of an effect of hMT + TMS on perceptual memory in our experiments suggests disruption of a memory trace that is dependent on the integrity of hMT + . An alternative interpretation, however, is that our TMS manipulation may affect transduction of an incoming sensory signal that elicits a memory trace further along the processing stream. Several factors, however, render this second interpretation less likely. First, interference with the incoming sensory signal is maximized by applying TMS at or close to the time of visual stimulation (Hotson et al., 1994; Silvanto et al., 2005). We stimulated in the 
blank intervals separating presentations and, moreover, found no reduction of our effects at longer blank durations, where the temporal gap between TMS and visual stimulation was up to 719 ms: well outside the effective range for disrupting motion processing directly (for review, see Laycock et al., 2007). In addition, interference with the sensory representation in hMT + might reasonably be expected to affect the percept of motion and depth ordering (Hotson et al., 1994; DeAngelis et al., 1998; Grunewald et al., 2002; Parker et al., 2002; Silvanto et al., 2005; Brouwer and van Ee, 2007), yet our data provide no indication of such a perceptual disruption-subjects continued to report their perception as quickly and consistently as in the other conditions (see Results). Other evidence from TMS studies of motion priming also suggests that disruption of incoming stimuli is insufficient to explain the effects of TMS on sensory memory because the processing of incoming stimuli themselves was unaffected (Campana et al., 2006). Thus, our present results indicate that hMT + TMS is more likely to directly disrupt perceptual memory for our ambiguous motion stimulus, providing evidence for hMT + as a cortical locus of this memory.

The proportion of percept repetition on pairs of consecutive presentations (Fig. 3) is an intuitive measure of perceptual memory that has been popular for some time (Leopold et al., 2002; Maier et al., 2003; Chen and He, 2004; Klink et al., 2008; Sterzer and Rees, 2008). We here introduced an additional, and in a sense complementary, measure of perceptual memory, namely the proportion of presentations for which the preferred percept is reported (Figs. 4, 5). In contrast to the proportion of percept repetition, this latter measure is particularly suitable for identifying changes in the gradual accumulation of perceptual memory across many presentations, thus putting to practical use recent reports that highlight the importance of such longer-term effects of perceptual memory (Maloney et al., 2005; Brascamp et al., 2008, 2009a; Pastukhov and Braun, 2008). We did not find any TMS effects on the former of these measures, the proportion of percept repetition on consecutive presentations (Fig. 3). This could indicate that hMT + is less involved in the faster-acting components of perceptual memory than in the slowly accumulating components probed with the latter measure (Fig. 5). At the same time, it is important to note a dual role of perceptual biases in this regard. On the one hand, the existence of perceptual biases allows us to identify changes in gradual memory accumulation, as this accumulation acts to attenuate the influence of these biases (Figs. 4, 5). On the other hand, these same biases limit the interpretability of the percept repetition measure on its own (Fig. 3), because an observer who is biased toward a particular interpretation will show a high proportion of percept repetition (namely of the preferred percept) even in the absence of perceptual memory. For instance, Carter and Cavanagh (2007) identified high proportions of percept repetition in their experiments as a sign of bias, not of perceptual memory. This consideration calls into question the most straightforward assumption that an attenuation of perceptual memory should always equal a reduction in percept repetition and, in general, provides a footnote to conclusions drawn from the percept repetition measure in isolation.

An interesting parallel exists between our findings and previous work by VanRullen et al. (2008) that showed an effect of TMS applied to the right parietal lobe on perception of the bistable "continuous wagon wheel illusion." In this illusion observers perceive a rotating stimulus (such as a wagon wheel) moving in the direction opposite to its actual motion for a portion of the viewing time. It was found that right-parietal TMS reduced the predominance of this illusory percept. As in our study, VanRullen et al. (2008) thus essentially reported an increased predominance of the preferred percept under TMS (in their case this was the veridical percept-in our experiment there was, of course, no veridical percept). This is a striking parallel that deserves notion, and that may prove of relevance in the future.

Existing ideas on perceptual memory suggest how TMS might influence this function on a neurophysiological level. Previous work has given rise to the view that perceptual memory may rely on an imbalance, brought about by prior perception, between the sensitivity states of neural populations that are associated with the alternative percepts (Noest et al., 2007; Wilson, 2007; Brascamp et al., 2008, 2009a). There is evidence that such populations exist in $\mathrm{hMT}+$ for the case of ambiguous rotation in depth (Grunewald et al., 2002; Parker et al., 2002; Brouwer and van Ee, 2007). TMS could thus affect perceptual memory in our study by attenuating this sensitivity imbalance, for instance by causing random activity in hMT +, thus effectively injecting "neural noise" (Walsh and Cowey, 2000). This idea is consistent with the fact that TMS over this area also reduces persistence of the motion aftereffect (Stewart et al., 1999; Théoret et al., 2002), another phenomenon thought to arise from a sensitivity imbalance between differently tuned neural populations (Mather, 1980; Grunewald and Lankheet, 1996).

\section{References}

Bisley JW, Zaksas D, Pasternak T (2001) Microstimulation of cortical area MT affects performance on a visual working memory task. J Neurophysiol 85:187-196.

Brascamp JW, Knapen TH, Kanai R, Noest AJ, van Ee R, van den Berg AV (2008) Multi-timescale perceptual history resolves visual ambiguity. PLoS One 3:e1497.

Brascamp JW, Pearson J, Blake R, van den Berg AV (2009a) Intermittent ambiguous stimuli: implicit memory causes periodic perceptual alternations. J Vis 9:3.1-23.

Brascamp JW, Kanai R, Walsh V, van Ee R (2009b) The influence of TMS over MT on perceptual memory in structure-from-motion rivalry [Abstract]. J Vis 9:8.263a.

Braunstein M (1977) Perceived direction of rotation of simulated threedimensional patterns. Percept Psychophys 21:553-557.

Brouwer GJ, van Ee R (2006) Endogenous influences on perceptual bistability depend on exogenous stimulus characteristics. Vision Res 46:3393-3402.

Brouwer GJ, van Ee R (2007) Visual cortex allows prediction of perceptual states during ambiguous structure-from-motion. J Neurosci 27: 1015-1023.

Campana G, Cowey A, Walsh V (2002) Priming of motion direction and area V5/MT: a test of perceptual memory. Cereb Cortex 12:663-669.

Campana G, Cowey A, Walsh V (2006) Visual area V5/MT remembers "what" but not "where". Cereb Cortex 16:1766-1770.

Carter O, Cavanagh P (2007) Onset rivalry: brief presentation isolates an early independent phase of perceptual competition. PLoS One 2:e343.

Chen X, He S (2004) Local factors determine the stabilization of monocular ambiguous and binocular rivalry stimuli. Curr Biol 14:1013-1017.

DeAngelis GC, Cumming BG, Newsome WT (1998) Cortical area MT and the perception of stereoscopic depth. Nature 394:677-680.

Drew AS, van Donkelaar P (2007) The contribution of the human FEF and SEF to smooth pursuit initiation. Cereb Cortex 17:2618-2624.

Dukelow SP, DeSouza JF, Culham JC, van den Berg AV, Menon RS, Vilis T (2001) Distinguishing subregions of the human MT + complex using visual fields and pursuit eye movements. J Neurophysiol 86:1991-2000.

Dumoulin SO, Bittar RG, Kabani NJ, Baker CL Jr, Le Goualher G, Bruce Pike G, Evans AC (2000) A new anatomical landmark for reliable identification of human area V5/MT: a quantitative analysis of sulcal patterning. Cereb Cortex 10:454-463.

Ellison A, Battelli L, Cowey A, Walsh V (2003) The effect of expectation on facilitation of colour/form conjunction tasks by TMS over area V5. Neuropsychologia 41:1794-1801.

Gagnon D, Paus T, Grosbras MH, Pike GB, O’Driscoll GA (2006) Transcra- 
nial magnetic stimulation of frontal oculomotor regions during smooth pursuit. J Neurosci 26:458-466.

Grunewald A, Lankheet MJ (1996) Orthogonal motion after-effect illusion predicted by a model of cortical motion processing. Nature 384:358-360.

Grunewald A, Bradley DC, Andersen RA (2002) Neural correlates of structure-from-motion perception in macaque V1 and MT. J Neurosci 22:6195-6207.

Hol K, Koene A, van Ee R (2003) Attention-biased multi-stable surface perception in three-dimensional structure-from-motion. J Vis 3:486-498.

Hotson J, Braun D, Herzberg W, Boman D (1994) Transcranial magnetic stimulation of extrastriate cortex degrades human motion direction discrimination. Vision Res 34:2115-2123.

Huk AC, Dougherty RF, Heeger DJ (2002) Retinal and functional subdivision of human areas MT and MST. J Neurosci 22:7195-7205.

Klink P, van Ee R, Nijs M, Brouwer G, Noest A, van Wezel, R (2008) Early interactions between neuronal adaptation and voluntary control determine perceptual choices in bistable vision. J Vis 8:16.1-16.18.

Kristjánsson Á (2006) Rapid learning in attention shifts: a review. Vis Cogn 13:324-362.

Laubrock J, Engbert R, Kliegl R (2008) Fixational eye movements predict the perceived direction of ambiguous apparent motion. J Vis 8:13.18.13.17.

Laycock R, Crewther DP, Fitzgerald PB, Crewther' SG (2007) Evidence for fast signals and later processing in human V1/V2 and V5/MT+: a TMS study of motion perception. J Neurophysiol 98:1253-1262.

Leopold DA, Wilke M, Maier A, Logothetis NK (2002) Stable perception of visually ambiguous patterns. Nat Neurosci 5:605-609.

Magnussen S, Greenlee MW (1999) The psychophysics of perceptual memory. Psychol Res 62:81-92.

Maier A, Wilke M, Logothetis NK, Leopold DA (2003) Perception of temporally interleaved ambiguous patterns. Curr Biol 13:1076-1085.

Maloney LT, Dal Martello MF, Sahm C, Spillmann L (2005) Past trials influence perception of ambiguous motion quartets through pattern completion. Proc Natl Acad Sci U S A 102:3164-3169.

Mather G (1980) The movement aftereffect and a distribution-shift model for coding the direction of visual movement. Perception 9:379-392.

McKeefry DJ, Burton MP, Vakrou C, Barrett BT, Morland AB (2008) Induced deficits in speed perception by transcranial magnetic stimulation of human cortical areas V5/MT+ and V3A. J Neurosci 28:6848-6857.

Noest A, van Ee R, Nijs M, van Wezel R (2007) Percept-choice sequences driven by interrupted ambiguous stimuli: a low-level neural model. J Vis 7:10.1-10.14.

Orbach J, Zucker E, Olson R (1966) Reversibility of the Necker cube. VII. Reversal rate as a function of figure-on and figure-off durations. Percept Motor Skills 22:615-618.

O'Shea J, Muggleton NG, Cowey A, Walsh V (2004) Timing of target discrimination in human frontal eye field. J Cogn Neurosci 16:1060-1067.

Parker A, Krug K, Cumming B (2002) Neuronal activity and its links with the perception of multi-stable figures. Philos Trans R Soc Lond Ser B Biol Sci 357:1053-1062.
Pasternak T, Greenlee M (2005) Working memory in primate sensory systems. Nat Rev Neurosci 6:97-107.

Pastukhov A, Braun J (2008) A short-term memory of multi-stable perception. J Vis 8:7.1-7.14.

Pearson J, Brascamp J (2008) Sensory memory for ambiguous vision. Trends Cogn Sci 12:334-341.

Pearson J, Clifford CG (2004) Determinants of visual awareness following interruptions during rivalry. J Vis 4:196-202.

Salzman CD, Murasugi CM, Britten KH, Newsome WT (1992) Microstimulation of visual area MT: effects on direction discrimination performance. J Neurosci 12:2331-2355.

Schacter DL, Buckner RL (1998) Priming and the brain. Neuron 20: $185-195$.

Schwartz O, Hsu A, Dayan P (2007) Space and time in visual context. Nat Rev Neurosci 8:522-535.

Silvanto J, Lavie N, Walsh V (2005) Double dissociation of V1 and V5/MT activity in visual awareness. Cereb Cortex 15:1736-1741.

Sterzer P, Kleinschmidt A (2007) A neural basis for inference in perceptual ambiguity. Proc Natl Acad Sci U S A 104:323-328.

Sterzer P, Rees G (2008) A neural basis for percept stabilization in binocular rivalry. J Cogn Neurosci 20:389-399.

Sterzer P, Russ MO, Preibisch C, Kleinschmidt A (2002) Neural correlates of spontaneous direction reversals in ambiguous apparent visual motion. Neuroimage 15:908-916.

Stewart L, Battelli L, Walsh V, Cowey A (1999) Motion perception and perceptual learning studied by magnetic stimulation. Electroencephalogr Clin Neurophysiol Suppl 51:334-350.

Théoret H, Kobayashi M, Ganis G, Di Capua P, Pascual-Leone A (2002) Repetitive transcranial magnetic stimulation of human area MT/V5 disrupts perception and storage of the motion aftereffect. Neuropsychologia 40:2280-2287.

VanRullen R, Pascual-Leone A, Battelli L (2008) The continuous wagon wheel illusion and the 'when' pathway of the right parietal lobe: a repetitive transcranial magnetic stimulation study. PLoS One 3:e2911.

Walker R, Techawachirakul P, Haggard P (2009) Frontal eye field stimulation modulates the balance of salience between target and distractors. Brain Res 1270:54-63.

Wallach H, O'Connell DN (1953) The kinetic depth effect. J Exp Psychol 45:205-217.

Walsh V, Cowey A (2000) Transcranial magnetic stimulation and cognitive neuroscience. Nat Rev Neurosci 1:73-79.

Walsh V, Ellison A, Battelli L, Cowey A (1998) Task-specific impairments and enhancements induced by magnetic stimulation of human visual area V5. Proc Biol Sci 265:537-543.

Wassermann EM (1998) Risk and safety of repetitive transcranial stimulation: report and suggested guidelines from the International Workshop on the Safety of Repetitive Transcranial Magnetic Stimulation, June 5-7, 1996. Electroencephalogr Clin Neurophysiol 108:1-16.

Wilson HR (2007) Minimal physiological conditions for binocular rivalry and rivalry memory. Vision Res 47:2741-2750. 